\title{
Experimental study of strain fields during shearing of medium and high-strength steel sheet
}

\author{
E. Gustafsson ${ }^{1 *}$ (D) L. Karlsson ${ }^{1}$ and M. Oldenburg ${ }^{2}$
}

\begin{abstract}
Background: There is a shortage of experimentally determined strains during sheet metal shearing. These kinds of data are a requisite to validate shearing models and to simulate the shearing process.

Methods: In this work, strain fields were continuously measured during shearing of a medium and a high strength steel sheet, using digital image correlation. Preliminary studies based on finite element simulations, suggested that the effective surface strains are a good approximation of the bulk strains below the surface. The experiments were performed in a symmetric set-up with large stiffness and stable tool clearances, using various combinations of tool clearance and clamping configuration. Due to large deformations, strains were measured from images captured in a series of steps from shearing start to final fracture. Both the Cauchy and Hencky strain measures were considered, but the difference between these were found negligible with the number of increments used (about 20 to 50).

Force-displacement curves were also determined for the various experimental conditions.
\end{abstract}

Results: The measured strain fields displayed a thin band of large strain between the tool edges. Shearing with two clamps resulted in a symmetric strain band whereas there was an extended area with large strains around the tool at the unclamped side when shearing with one clamp. Furthermore, one or two cracks were visible on most of the samples close to the tool edges well before final fracture.

Conclusions: The fracture strain was larger for the medium strength material compared with the high-strength material and increased with increasing clearance.

Keywords: Sheet metal, Experiment, Shearing, Force, Strain, Strain field

\section{Background}

Shearing is a widely used process in the sheet metal industry and has been extensively studied to increase the understanding of the shearing mechanism, but also to improve the industrial process with respect to parameters such as tool force, tool wear and sheared surface appearance. Tool forces were measured for various materials already by Izod (1906), who used a symmetric set-up with double tools to obtain a large stiffness in the lateral direction and a stable clearance. A refined set-up was devised by Gustafsson et al. (2014) which allowed the measurement of the lateral force component (the component that strives

*Correspondence: egu@du.se

'Dalarna University, SE-791 88 Falun, Sweden

Full list of author information is available at the end of the article to separate the tools and increase the clearance), in addition to the force in the shearing direction, with a minimal reduction of lateral stiffness. The lateral force was previously measured by Yamasaki and Ozaki (1991) but with friction losses in sliding guides and a low stiffness in the set-up that results in tool clearance changes during shearing. The same concept was improved by Kopp et al. (2016) for increased stiffness and with compensation of friction losses through calibrations. Still, the stiffness is lower than in symmetric double shearing and there is a significant risk of misinterpreted forces due to changed conditions in the sliding guides.

Closely related to shearing is blanking, a process which has an inherent clearance stability due to its rotational symmetric set-up. The appearance of the cut surfaces in the blanking process was studied by Crane (1927), who 
characterised the surface appearance in terms of various zones. Crane (1927) also conducted post-shearing tensile tests to relate the surface appearance with the deformation and fracture characteristics in the tensile testing. The properties of the sheared sheet is also influenced by the properties of the plastically deformed material in a region beneath the cut sheet surface. Micro-hardness testing of sheared samples was used by Weaver and Weinmann (1985) to study the deformation hardening in this region, and Dalloz et al. (2009) showed that a heavily deformed sub-surface region contains crack initiating voids.

The strains which arise in the sheet metal during shearing influence the properties of the sheared sheet. Furthermore, experimentally measured strains are a requisite for validation of newly developed shearing models, especially for initiation of fracture. Digital image correlation (DIC) is an established and frequently used technique for non-contact strain measurement. The fundamentals of DIC is covered by, for example, Sutton et al. (2009). This technique was used to study the local deformation during planar blanking by Stegeman et al. (1999), and by Tarigopula et al. (2008) to measure large plastic deformation during shear testing of dual phase steel. A method in which the metal flow line tilting angle was measured and converted to local shear strain, was used by Wu et al. (2012) to characterise the strain distribution within mechanically sheared edges of dual phase steels.

In this study the DIC-technique was used to evaluate the strain distribution on sheared sheets of a medium and a high strength steel, using various clearances and clamp configurations. The strain fields are presented at particular stages of tool penetration corresponding to a fixed tool displacement, crack initiation and final fracture. In addition to the strain fields, the force-displacement curves were determined for the various experimental conditions. Furthermore, a generic finite element simulation was done to compare a plain strain assumption with an actual three dimensional state at the surface, and hence, evaluate if surface strains can be used to approximate the bulk strains.

\section{Finite element simulations}

Finite element (FE) simulations were applied to compare the strains on the sheet surface and the interior of the sheet. If there is a good agreement between these strain fields, then a strain field measured on the sheet surface can be used also as a measure of the strains within the sheet. This would increase the relevance of the surface strain measurements since most of the shearing is of bulk material.

\section{Model}

FE analyses of shearing, modelled in both two dimensional (2D) plane strain and three dimensions (3D), were performed with a commercial general-purpose finite element software. Geometry and boundary conditions for the 2D plane strain model are described in detail by Gustafsson et al. (2014). The same conditions were applied to the 3D model. Both models were coarsely meshed except in the area where large deformations were expected and a denser mesh were required to resolve gradients in the state variables. Four-noded and fully integrated plane strain elements were used in the 2D model and eightnoded fully integrated selective reduced solid elements were used in the 3D model. Adaptive remeshing was used for the 2D model. Contacts were modelled with surface to surface formulations and both static and dynamic friction coefficients were 0.1 .

Elastic tools and an isotropic elastic-plastic sheet material were used in both models. Poisson's ratio was 0.3 and Young's modulus was $210 \mathrm{GPa}$ for all materials. The yield stress for the sheet material was described with an exponential hardening law according to Hollomon (1945), $\sigma_{Y}=K \bar{\varepsilon}_{p}^{n}$, where $\bar{\varepsilon}_{p}$ is the effective plastic strain and $K$ and $n$ are material specific parameters.

A constant tool acceleration of $100 \mathrm{~mm} \mathrm{~s}^{-2}$ was used in all simulations.

\section{Strain comparison}

Effective strains from FE simulations at the surface of a 3D simulation, the interior of a 3D simulation, and at plane strain are shown in Fig. 1. Due to element distortion in the 3D model, where no adaptive remeshing was applied, the strain field comparison was done at moderate tool displacement. The similar appearance of the strain fields in Fig. 1 suggest that the effective strain on the surface and inside the material are comparable. Plane strain was earlier shown to be a good approximation in terms of shearing forces by Gustafsson et al. (2014) for parallel tools and also for angled tools by Gustafsson et al. (2016a). This observation implies that strains on the sheet surface are representative for the interior strains and that plane strain simulations are once again a good approximation.

\section{Measures of strain}

Rate of deformation is the only strain rate measure that is energetically conjugate with the Cauchy stress. In a Cartesian coordinate system, the rate of deformation tensor is

$$
D_{i j}=\frac{1}{2}\left(v_{i, j}+v_{j, i}\right),
$$

where $v_{i, j}$ is the velocity gradient tensor written

$$
v_{i, j}^{2 \mathrm{D}}=\left[\begin{array}{ll}
\frac{\partial v_{1}}{\partial x_{1}} & \frac{\partial v_{1}}{\partial x_{2}} \\
\frac{\partial v_{2}}{\partial x_{1}} & \frac{\partial v_{2}}{\partial x_{2}}
\end{array}\right]=\left[\begin{array}{ll}
\frac{\partial v_{x}}{\partial x} & \frac{\partial v_{x}}{\partial y} \\
\frac{\partial v_{y}}{\partial x} & \frac{\partial v_{y}}{\partial y}
\end{array}\right],
$$

for two dimensions. True (logarithmic) strain is related to rate of deformation as 

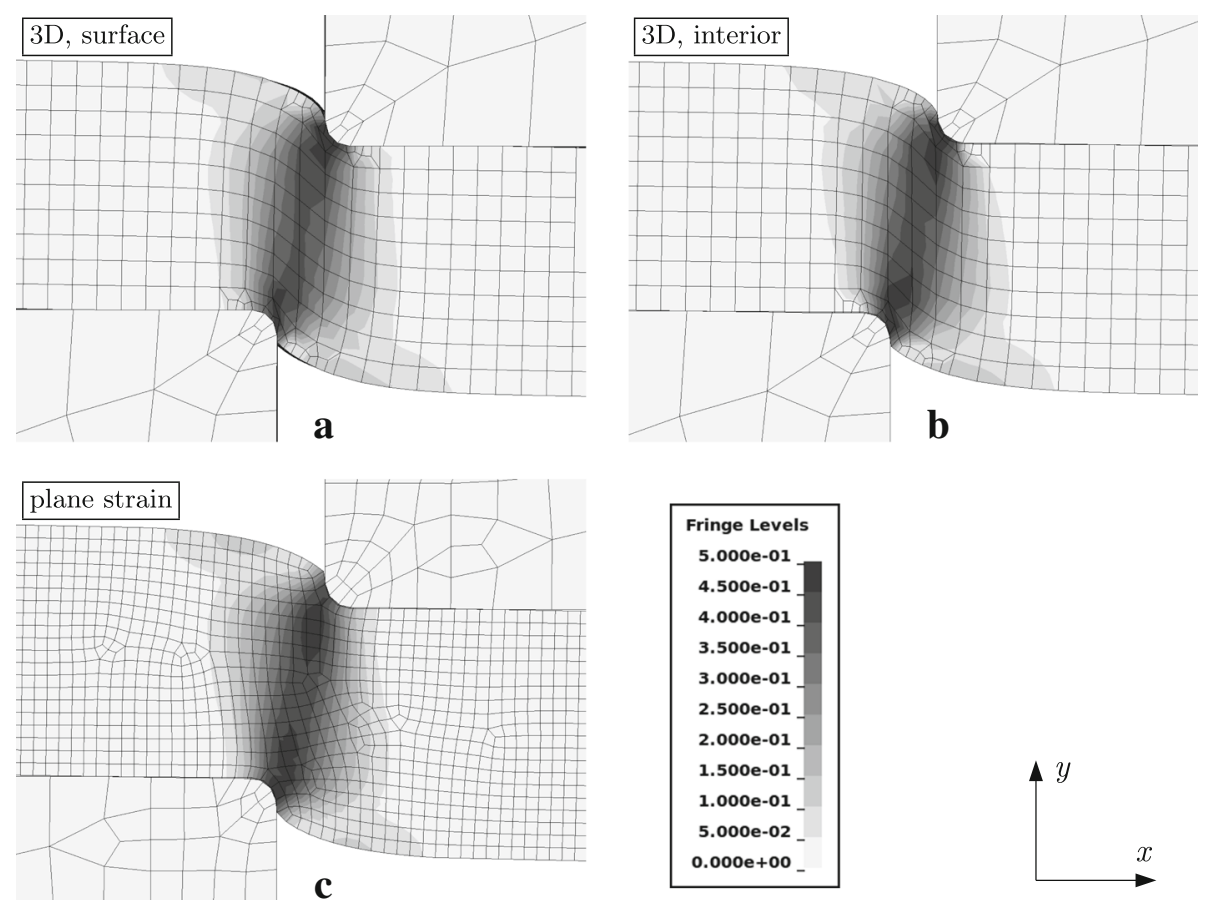

Fig. 1 Fringe plots of effective strain from $\mathbf{a}$ the surface of a 3D FE simulation, $\mathbf{b}$ inside the sheet of the same 3D simulation, and $\mathbf{c}$ a plane strain FE simulation. The plots were obtained at equal tool displacement $\left|U_{y}\right|$

$$
\dot{\varepsilon}_{i j}^{\text {True }}=D_{i j}
$$

If rigid body rotations are present, the strain tensor may be more easily interpreted if expressed in the original orientation. In the original orientation the strain is

$$
\dot{\varepsilon}_{i j}^{\prime \text { True }}=R_{m i} D_{m n} R_{n j}
$$

where $R_{i j}$ is the rotation tensor. The rotation does not alter the strain levels and thus the effective strain,

$$
\dot{\varepsilon}_{\mathrm{eff}}=\sqrt{\frac{2}{3} \dot{\varepsilon}_{i j} \dot{\varepsilon}_{i j}},
$$

is unaffected.

When the displacements originate from a range of images taken of the deformation process, the continuous rate of deformation is unknown and the best approximation is a mean rate of deformation between two images. In that case it is more convenient to describe deformation in terms of a displacement gradient tensor $u_{i, j}$, for each interval between two images, instead of a mean velocity gradient tensor. In two dimensions

$$
u_{i, j}^{2 \mathrm{D}}=\left[\begin{array}{ll}
\frac{\partial u_{1}}{\partial x_{1}} & \frac{\partial u_{1}}{\partial x_{2}} \\
\frac{\partial u_{2}}{\partial x_{1}} & \frac{\partial u_{2}}{\partial x_{2}}
\end{array}\right]=\left[\begin{array}{ll}
\frac{\partial u_{x}}{\partial x} & \frac{\partial u_{x}}{\partial y} \\
\frac{\partial u_{y}}{\partial x} & \frac{\partial u_{y}}{\partial y}
\end{array}\right] .
$$

The Cauchy strain tensor for one increment is then

$$
\Delta \varepsilon_{i j}^{\text {Cauchy }}=\frac{1}{2}\left(u_{i, j}+u_{j, i}\right)
$$

and, if the increments are small enough, the sum over a number of increments is a good approximation of the true strain. The logarithmic Hencky strain for each increment may result in an even better approximation, but due to an unknown deformation path inside the increment, it is still an approximation. Hencky strain and the rotation tensor is obtained through a polar decomposition of the deformation gradient,

$$
F_{i j}=\delta_{i j}+u_{i, j}
$$

where $\delta_{i j}$ is the Kronecker delta, into a rotation part and a stretch part $F_{i j}=R_{i k} U_{k j}$. The decomposition is accomplished by finding the eigenvalues $\lambda_{(n)}^{2}$ and normalised eigenvectors $N_{i}^{(n)}$ to the eigenvalue problem

$$
\left(C_{i j}-\lambda^{2} \delta_{i j}\right) N_{i}=0,
$$

where

$$
C_{i j}=F_{k i} F_{k j}
$$

is the right Cauchy-Green deformation tensor. Then, $\lambda_{(n)}$ are the principal stretches that act parallel to the $N_{i}^{(n)}$ directions. The number of eigenvalues and principal stretches $n_{D}$ depends on the number of dimensions in the calculations; if the problem is two dimensional then $n_{D}=2$. Further, the right stretch tensor is 


$$
U_{i j}=\sum_{n=1}^{n_{D}} \lambda_{(n)} N_{i}^{(n)} N_{j}^{(n)}
$$

and

$$
R_{i j}=F_{i k} U_{k j}^{-1},
$$

where $U_{i j}^{-1}$ is the inverse of the right stretch tensor. The inverse of a tensor, in this case $U_{i j}$, can be calculated as

$$
U_{i j}^{-1}=\frac{1}{2 \epsilon_{r s t} U_{r 1} U_{s 2} U_{t 3}} \epsilon_{j m n} \epsilon_{i p q} U_{m p} U_{n q},
$$

where $\epsilon_{i j k}$ is the Levi-Civita permutation called alternating tensor. The Hencky strain for the increment is therefore

$$
\Delta \varepsilon_{i j}^{\text {Hencky }}=\sum_{n=1}^{n_{D}} \log \lambda_{(n)} N_{i}^{(n)} N_{j}^{(n)},
$$

or rotated to the original configuration

$$
\Delta \varepsilon_{i j}^{\prime \text { Hencky }}=R_{m i} \varepsilon_{m n} R_{n j} \text {. }
$$

Finally, the incremental effective strain is

$$
\Delta \bar{\varepsilon}=\sqrt{\frac{2}{3} \Delta \varepsilon_{i j} \Delta \varepsilon_{i j}}
$$

where $\Delta \varepsilon_{i j}$ is either the Cauchy or the Hencky strain tensor. These increments are summed to obtain accumulated effective strain.

\section{Methods}

Strains on the sheet $x y$-surface (labelled image area in Fig. 2) were obtained after a number of steps that can be summarised in: sample preparation, shearing of speckled sheet samples under high speed photography, digital image correlation, strain calculations and finally creating images to show the strain levels. The steps are explained further in the following subsections.

\section{Materials and sample preparation}

Two materials were used in the study: a medium strength (MS) construction steel, and a high strength (HS) wear plate steel. The mechanical properties are shown in Table 1 and the stress-strain curves in Fig. 3.

Samples with outer dimensions of $140 \times 150 \mathrm{~mm}$ and a centred square, $100 \times 100 \mathrm{~mm}$, hole were laser cut and machined from the sheet (Fig. 2). The outer $x y$ surface of the samples was prepared with high contrast random speckles at a characteristic size of a few image pixels. Abrasive blasting was applied to the surface and the rugged surface was sprayed with dull black paint. The surface was then ground with a fine abrasive paper to remove the paint on the most protruding parts of the steel surface to create a high contrast speckle pattern as shown in Fig. 4. Use of the traditional fine spray of dull black paint over a dull white coat was not applicable in this experiment due to limited adhesion and large shear strains in the sheet.

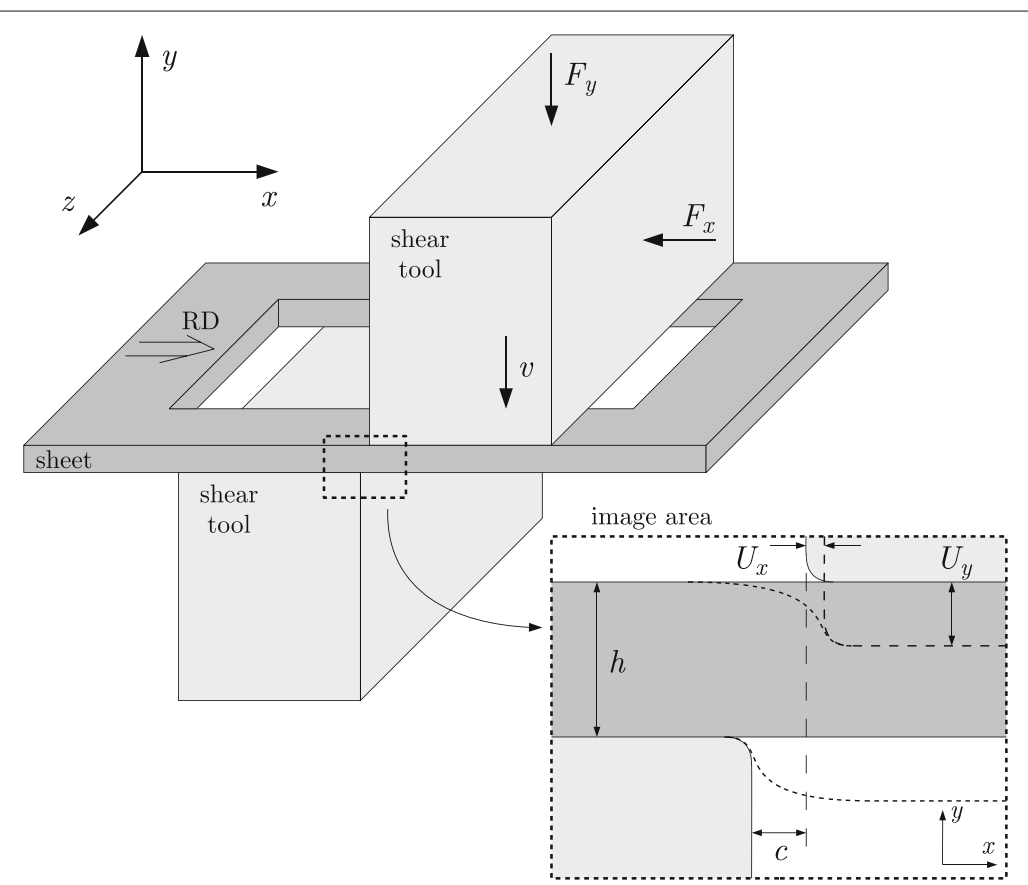

Fig. 2 Schematic representation of the rectangular sheet sample, with square hole, and tools together with definition of the coordinate system used. Reaction forces on the moving tool as result of the velocity $v$ are $F_{x}$ and $F_{y}$. Definitions of sheet thickness $h$, tool clearance $c$, and tool displacements $U_{x}$ and $U_{y}$ are shown in the magnified area. A speckle pattern was applied to the sheet $x y$-surface in the indicated image area. $R D$ indicates the rolling direction 
Table 1 Mechanical properties, evaluated from uniaxial tensile tests, of sheet metal grades used in the study. The thickness range of the sheared samples is also shown

\begin{tabular}{lllll}
\hline $\begin{array}{l}\text { Material } \\
\text { strength }\end{array}$ & $\begin{array}{l}\text { Yield strength, } \\
\mathrm{Rp} 02[\mathrm{MPa}]\end{array}$ & $\begin{array}{l}\text { Tensile strength, } \\
\mathrm{Rm}[\mathrm{MPa}]\end{array}$ & $\begin{array}{l}\text { Elongation, } \\
\mathrm{A} 80[\%]\end{array}$ & $\begin{array}{l}\text { Thickness } \\
{[\mathrm{mm}]}\end{array}$ \\
\hline Medium & 450 & 520 & 25 & $5.97-6.03$ \\
High & 1080 & 1260 & 7 & $6.11-6.15$ \\
\hline
\end{tabular}

\section{Shearing experiments}

The rectangular sheet samples were sheared in a set-up with two parallel tools as schematically shown in Fig. 2. The speckle pattern on the sheet surface was imaged by a camera during the entire shearing process. Accurate measurements of tool displacements and shearing forces, and also stable tool clearances, were provided by the symmetric set-up illustrated in Fig. 5. Details on the tool displacement measurements are covered by Gustafsson et al. (2016a). The initial development of the symmetric experimental set-up, including details of the force measurements, are described by Gustafsson et al. (2014).

The various combinations of clearances and clamping configurations which were used for shearing the sheets are shown in Table 2. Two experiments were performed for each indicated combination in Table 2.

\section{High-speed photography and image analysis}

A camera equipped with a lens with $200 \mathrm{~mm}$ focal length, was used to obtain an image of the speckle pattern within an image area of $15 \times 12 \mathrm{~mm}$ (see also Fig. 2). Images with a resolution of $1280 \times 1024$ pixels where captured at $1 \mathrm{kHz}$ with about $200 \mu \mathrm{s}$ exposure time. At this resolution and sample rate, the camera allowed approximately $1.6 \mathrm{~s}$ long image sequences which were sufficient for the shearing

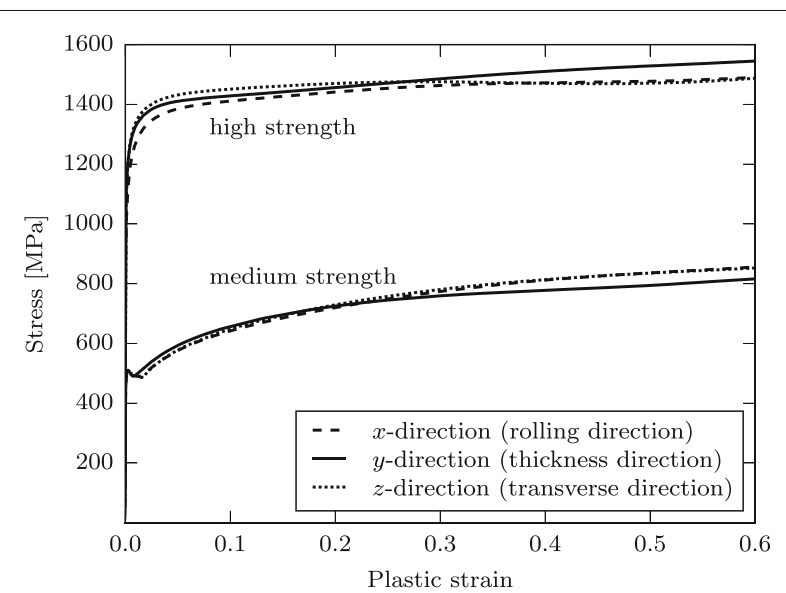

Fig. 3 Flow stress curves from uniaxial compression tests by Gustafsson et al. (2016b) in three directions for the two material grades. The $x-, y-$, and $z$-directions are defined in Fig. 2 process. A total of about 1600 images were hence captured for each shearing experiment (combination of material grade, clearance and clamping configuration). From each set of 1600 images, about 20 to 50 images were selected for further analysis as described in the next paragraph. The images were selected at regular intervals corresponding to a tool displacement of about 10 pixels $(\sim 0.1 \mathrm{~mm})$, so the number of images for analysis depended on the tool displacement before fracture. Most of the selected images were from the last part of the 1600 images since the tool velocity increased from shearing start to final fracture. The maximum camera speed was only needed for the final part of the shearing. Due to the distinct final fracture, and the following rapid tool displacement, the image sequences was easily manually synchronised in time to the recorded tool displacements and forces.

Digital image correlation (DIC) was applied to the images that were selected as described above. In this technique, two images are compared and analysed using image analysis algorithms to compute parameters such as the displacements of a solid body. For a detailed account of DIC, the reader is referred to a textbook on this topic, such as Sutton et al. (2009). In short, points in the first image are located in the second image by correlation of a surrounding rectangular area of eligible size called interrogation window and returned as a displacement field. The number of vectors in the displacement field equals the number of selected points for analysis. Here, the analysed points were positioned in an equally spaced rectangular matrix, eight pixels apart (approximately $0.1 \mathrm{~mm}$ on the sheet sample). The imaged object should be prepared with a random speckle pattern of appropriate speckle size so that each interrogation window contain a unique image.

Because of the large deformations, the images were analysed consecutively $(1+2,2+3,3+4, \ldots)$ which resulted in incremental displacement fields. The application if the DIC technique was performed with the open source JPIV software (JPIV r24, available under terms of GNU GPL, http://www.jpiv.vennemann-online.de).

The resulting displacement fields were used to calculate strain fields according to the following subsection.

\section{Evaluation of strain}

The displacement fields were used to calculate the effective strain (Eq. (16)) through either the Cauchy strain tensor (Eq. (7)) or the Hencky strain tensor (Eq. (14)). The strain tensor was calculated for each increment and the effective strain for each increment was added to the previous accumulated strain in material coordinates (Lagrange). The pixel size in the images of the strain fields was about $0.1 \mathrm{~mm}$. In these images, strains were shown as mean values over $3 \times 3$ pixels which means that the effective length of measurement was about $0.3 \mathrm{~mm}$. 


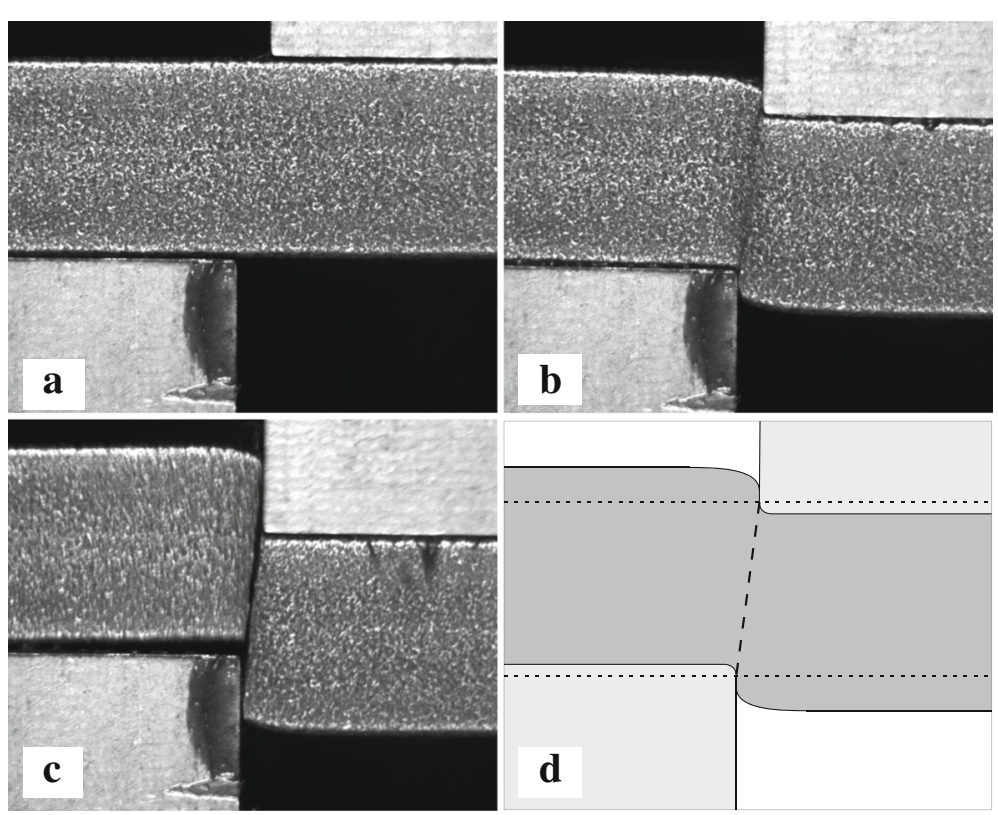

Fig. 4 Typical speckle pattern on the sheet strip xy-surface: a before shearing start, b just before final fracture, c final fracture immediately after b. The images were captured by the high-speed camera when shearing the high strength material at $0.15 \mathrm{~h}$ clearance and with two clamps. Note that the dark area on the lower tool is spilled grease and no tool defect. Panel $\mathbf{d}$ shows a schematic figure of the shearing. Strains were plotted along the thick dashed line and the largest strain found between the two thinner horizontal dashed lines was tabulated

A comparison between accumulated effective strain fields obtained from the Cauchy strain tensor and the Hencky strain tensor is presented in the results section. Since there was no apparent difference between the strain fields, only the more commonly used Cauchy strain is presented later in the paper. The accumulated strain fields are presented at various stages of the shearing to allow comparison of various parameters. The strain field images are an efficient tool to visualise the appearance of the strain distribution, but the actual strain value is often difficult to read from the fringe plots. Therefore, the strains on a straight line between the lower and the upper tool edges as well as the maximum strain in the shear area were evaluated. The line was positioned with its ends at the transition between the curved and the vertical tool contours as schematically shown in Fig. $4 \mathrm{~d}$. The maximum strains were restricted to the area between the two horizontal lines shown in Fig. 4d.

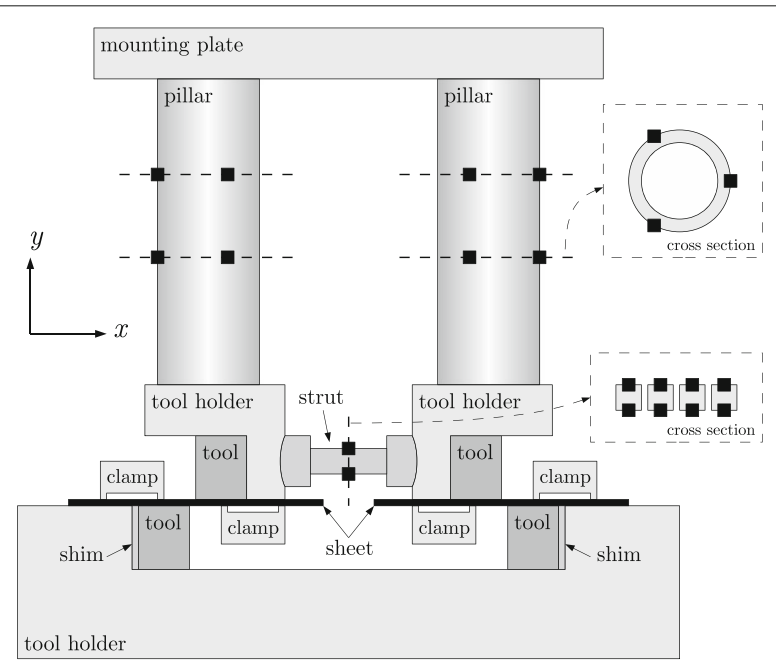

Fig. 5 Schematic front view of the experimental set-up showing strain gauge positions as black squares (Gustafsson et al. 2014). Sheet strips are clamped on both sides 
Table 2 Combinations of clearances, material grades and clamping configurations used for shearing the sheet samples. The tool clearance is expressed as a fraction of the sheet thickness $h$

\begin{tabular}{lllllll}
\hline & \multicolumn{2}{l}{ Medium strength } & & \multicolumn{2}{l}{ High strength } \\
\cline { 2 - 3 } Clearance & 1 clamp & 2 clamps & & 1 clamp & 2 clamps \\
\hline $0.05 h$ & $x$ & $x$ & & & \\
$0.15 h$ & $x$ & $x$ & & $x$ & $x$ \\
$0.25 h$ & $x$ & $x$ & & $x$ & $x$ \\
\hline
\end{tabular}

\section{Results}

\section{Shearing experiments}

Forces, $F_{x}$ and $F_{y}$, versus tool displacement, $\left|U_{y}\right|$, from the shearing experiments on the medium strength and high strength materials are shown in Fig. 6. The forcedisplacement curves are presented to indicate the range of forces and displacements for which strains were evaluated (see following subsections for these results). Among

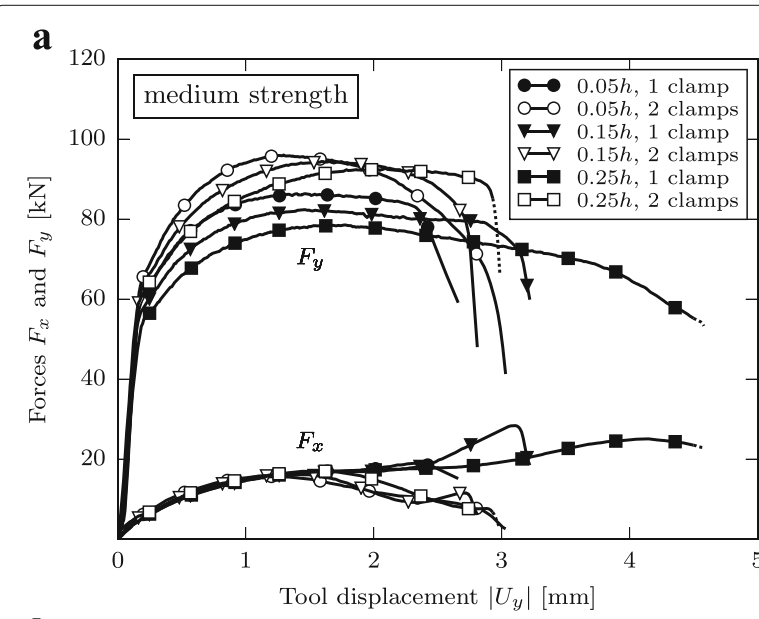

b

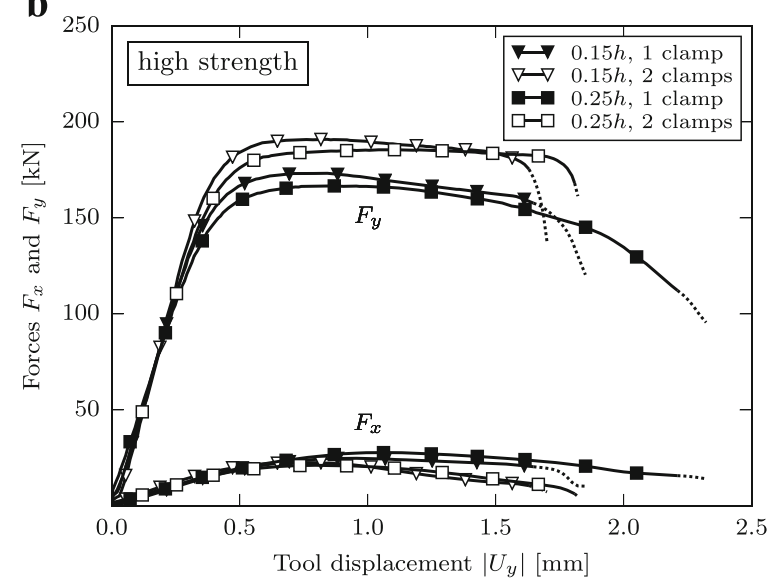

Fig. 6 Force-displacement curves from shearing of medium strength material (a) and high-strength material (b). Clearances (expressed as fractions of sheet thickness $h$ ) and clamping configurations are indicated in the figure legend. The dashed part of a curve indicates that it was impossible to image the speckle pattern on the sheet the two symmetric sides of the set-up and the two experiments performed for each configuration, the difference in force was less than $1 \%$ and $\left|U_{y}\right|$ varied a few percent at the point of fracture. These variations were smaller than the expected accuracy in the strain measurements, and therefore, strains were only calculated from one series of images for each combination of shearing parameters and material. Strains were evaluated from shearing start to short before fracture, that is, during practically the whole shearing process. For some samples, however, the shearing velocity was to large to allow sharp image capturing and strain evaluation. In those cases it was impossible to record images during the later stages of the shearing, as indicated by the dotted force-displacement curves in Fig. 6.

\section{Comparison of strain measures}

There was no obvious visual difference between the Cauchy strain and the Hencky strain, as exemplified in Fig. 7. If, however, the difference $\bar{\varepsilon}$ Cauchy $-\bar{\varepsilon}^{\text {Hencky }}$ was calculated, then a difference between the strain measures of up to about 0.2 was revealed as shown in Fig. 7e and $\mathrm{f}$. This is still a small difference compared with the strain levels: the strain difference 0.2 corresponds to a relative difference of about $7 \%$. The distribution of the differences seemed random. All other material grades, clearances and clamp configurations displayed differences in the same range as shown in Fig. 7e, f.

\section{Strain field measurements}

Accumulated strains over the shearing cycle from start to short before fracture, as indicated by the end of the solid lines in Fig. 6, are shown for the medium and highstrength materials in Figs. 8 and 9, respectively. Most of the strain fields showed a thin band of large strain across the sheet. The strain band across the sheet was most straight for the $0.15 h$ clearances; the curvature increased for both smaller and larger clearances. With two clamps the strain band was mostly symmetric. Shearing with one clamp resulted in a large strain concentration at the lower tool and a wider strain band as compared with shearing with two clamps. The extra width of the strain band appeared on the unclamped side of a line between the tool edges. In general, the strains before final fracture were larger in the medium strength material compared with the high strength material. Most samples had one or two visible cracks at the tool edges well before final fracture. At $0.05 h$ clearance and with two clamps, shown in Fig. 8b, the cracks propagated so that they circumvent each other with large deformation in the material between the cracks before final fracture as result.

Strains on a straight line from the lower to the upper tool edge, as defined in Fig. 4d, were evaluated and plotted in Fig. 10. The line is indicated in the sheared samples by the dashed lines in Figs. 8 and 9. While the strain levels were 

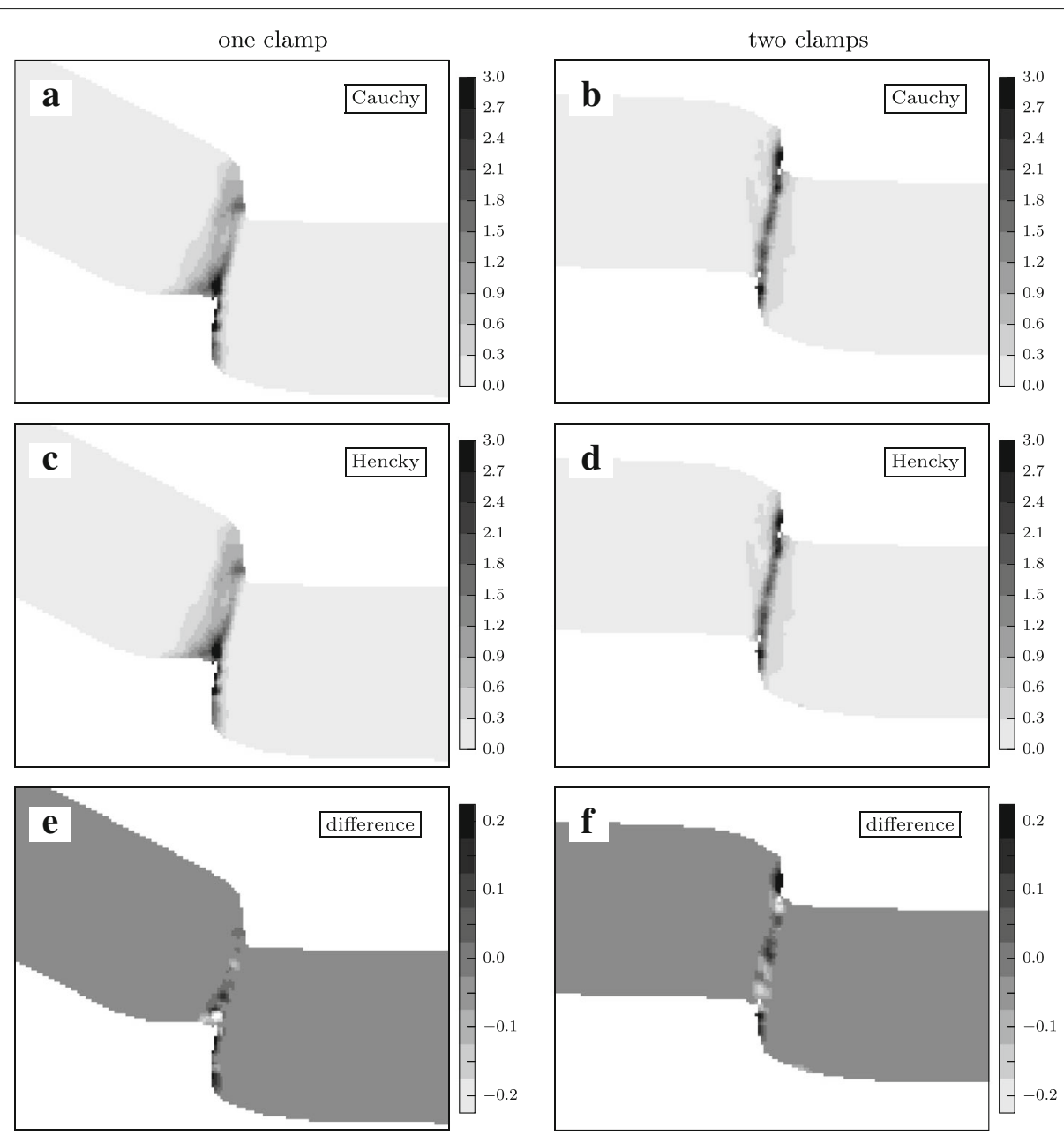

Fig. 7 Comparison of Cauchy and Hencky strain measures for the medium strength material and 0.15h clearance using one clamp (left panels) and two clamps (right panels) right before fracture. The difference shown in panels $\mathbf{e}$ and $\mathbf{f}$ was calculated as $\bar{\varepsilon}^{\text {Cauchy }}-\bar{\varepsilon}^{\text {Hencky }}$

easy to interpret, the line gave little information of the strain distribution. In case of one clamp (Fig. 10a, c), the strain concentration at the lower tool was captured, but the line then continues mostly outside of the band with elevated strain levels. With two clamps (Fig. 10b, d) the line mostly coincides with the high strain band, especially for the $0.05 h$ and $0.15 h$ clearances. At $0.25 h$ clearance the strain band was more s-shaped and a peak on the strain curves was shown at each of the multiple intersections between the line and the strain band. Part of the curves, in the beginning or end of the line, may be missing if the material had a visible crack underneath as, for example, the beginning of the curve for $0.15 h$ clearance in Fig. 10c.

The medium strength material sheared at $0.05 \mathrm{~h}$ clearance with two clamps showed large strains in an area about halfway between the lower and upper tool edges (Fig. 10b). This was due to the observed behaviour where cracks propagate from each tool so that they wrapped around each other as seen in Fig. 8b. The remaining material between the cracks is sheared to a much larger tool displacement $\left|U_{y}\right|$ and thus larger strains compared with the earlier fractured material at the sheet $x z$-surfaces. Also compared with samples sheared at larger clearance, where the cracks are more prone to meet in the middle, the strain at final fracture is much larger (compare Fig. 8b, $\mathrm{d}$, and $\mathrm{f}$ ).

The maximum strains, $\bar{\varepsilon}_{\text {final }}$, were determined and are shown in Table 3 together with corresponding tool displacement $\left|U_{y}\right|_{\text {final }}$. The positions of the maximum strains are indicated by white circles in Figs. 8 and 9. For all experimental conditions in Figs. 8 and 9, except Figs. 8e and 9c $(0.25 h$ clearance and one clamp for both material strength levels), the position of the maximum strain fell, approximately, on the line between the tool edges along where strains were evaluated.

When there was a visible crack before the final fracture, also the strains at crack initiation are of interest to compare. Therefore, the maximum strains at the first 

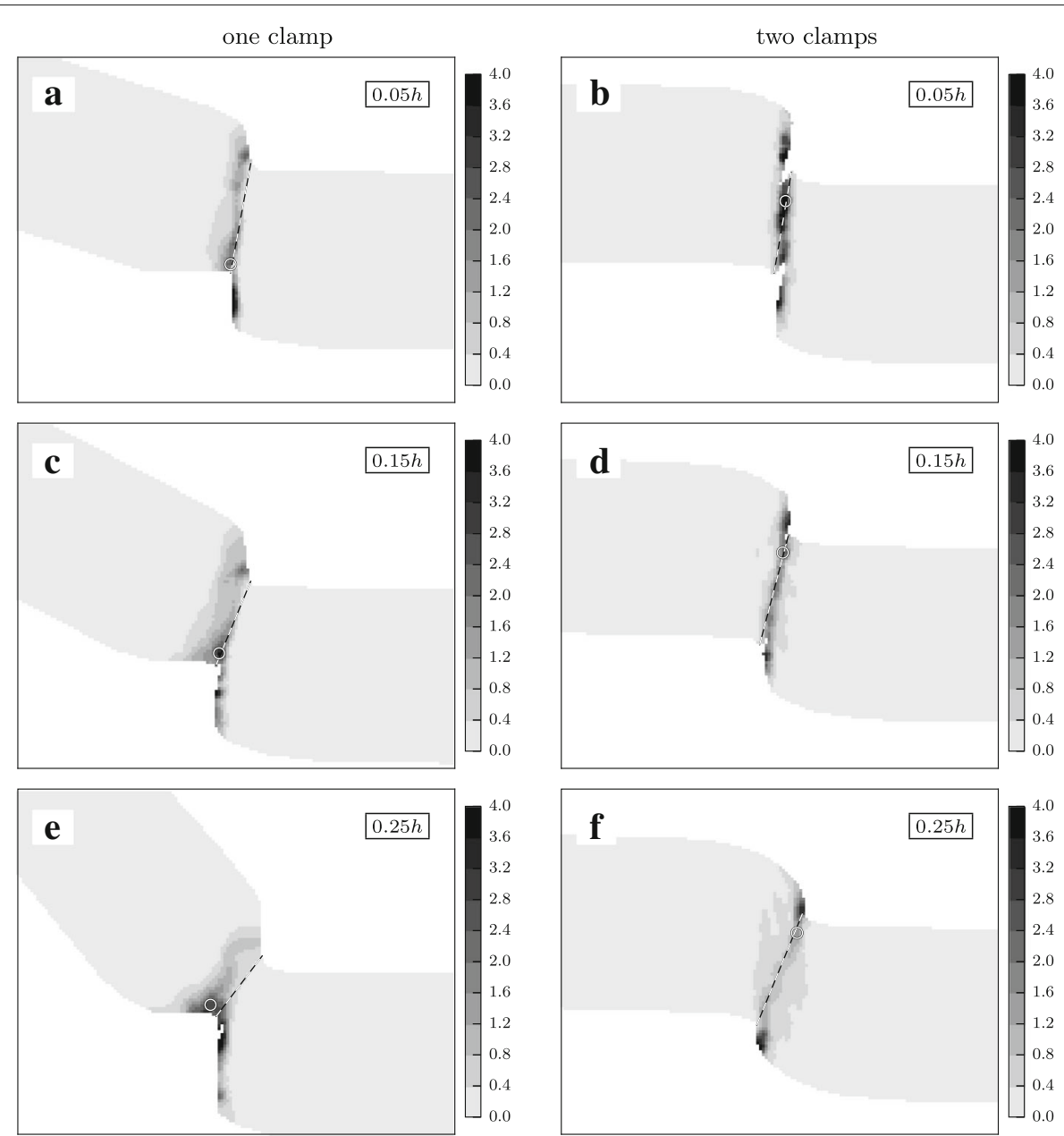

Fig. 8 Effective Cauchy strain fields (Eqs. 5 and 7) short before final fracture from shearing of the medium strength material using one clamp (left panels) and two clamps (right panels). Clearances, expressed as fractions of sheet thickness $h$, are indicated in each panel. Strains along the dashed black and white line are plotted in Fig. 10. The positions of the maximum strains in the shear bands are indicated by white circles, and this maximum strain is shown in Table 3

appearance of a crack on the imaged sheet surface (crack initiation) and the corresponding applied tool displacements are shown in Table 3 as $\bar{\varepsilon}_{\text {init }}$ and $\left|U_{y}\right|_{\text {init }}$, respectively. Strain fields and line plots for these tool displacements are shown in Figs. 11, 12 and 13, in the same way as earlier shown at final fracture. Here, the maximum strain is located close to the drawn line for all combinations of materials and shearing parameters, as shown by the dashed lines and white circles in Figs. 11 and 12 . When the high strength material was sheared with two clamps and $0.25 h$ clearance the lapse from crack initiation to fracture was very short. Initiated cracks were hard to distinguish from the preceding narrow shear bands, which made the exact moment of crack initiation, and thus also $\bar{\varepsilon}_{\text {init }}$ in Table 3 , somewhat hard to determine. Overall, the fracture strains were larger for the medium strength material compared with the high strength material, and increased with increased tool clearance. With two clamps, however, the maximum strain at final fracture, $\bar{\varepsilon}_{\text {final }}$, decreased with increased tool clearance.

With exception of $\bar{\varepsilon}_{\text {final }}$ for the medium strength material, $0.05 h$ with two clamps, and $0.25 h$ with one clamp, the maximum strains presented in Table 3 are at such positions on the sheets where they are comparable to the strain at the crack tip and thus they are approximations of the fracture strain. The fracture strains are therefore larger at the final fracture compared with at crack initiation.

Figures 8, 9, 11, and 12 show strain at various tool displacements for all combinations of shearing parameters. A comparison of the strains at a given tool displacement, same for all combinations of shearing parameters, can also be of interest. The sample with the least tool displacement at fracture was the high strength material at $0.15 \mathrm{~h}$ 

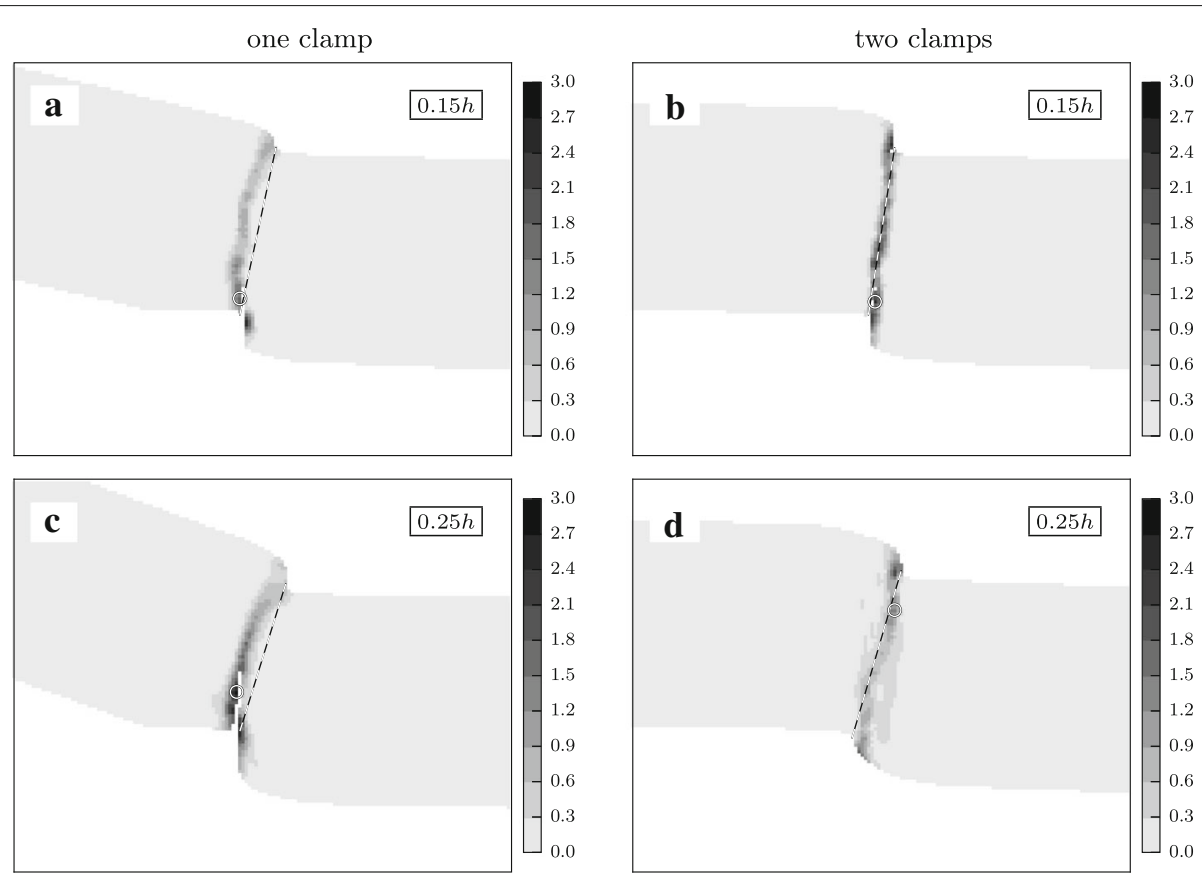

Fig. 9 Same as Fig. 8 but for the high-strength material

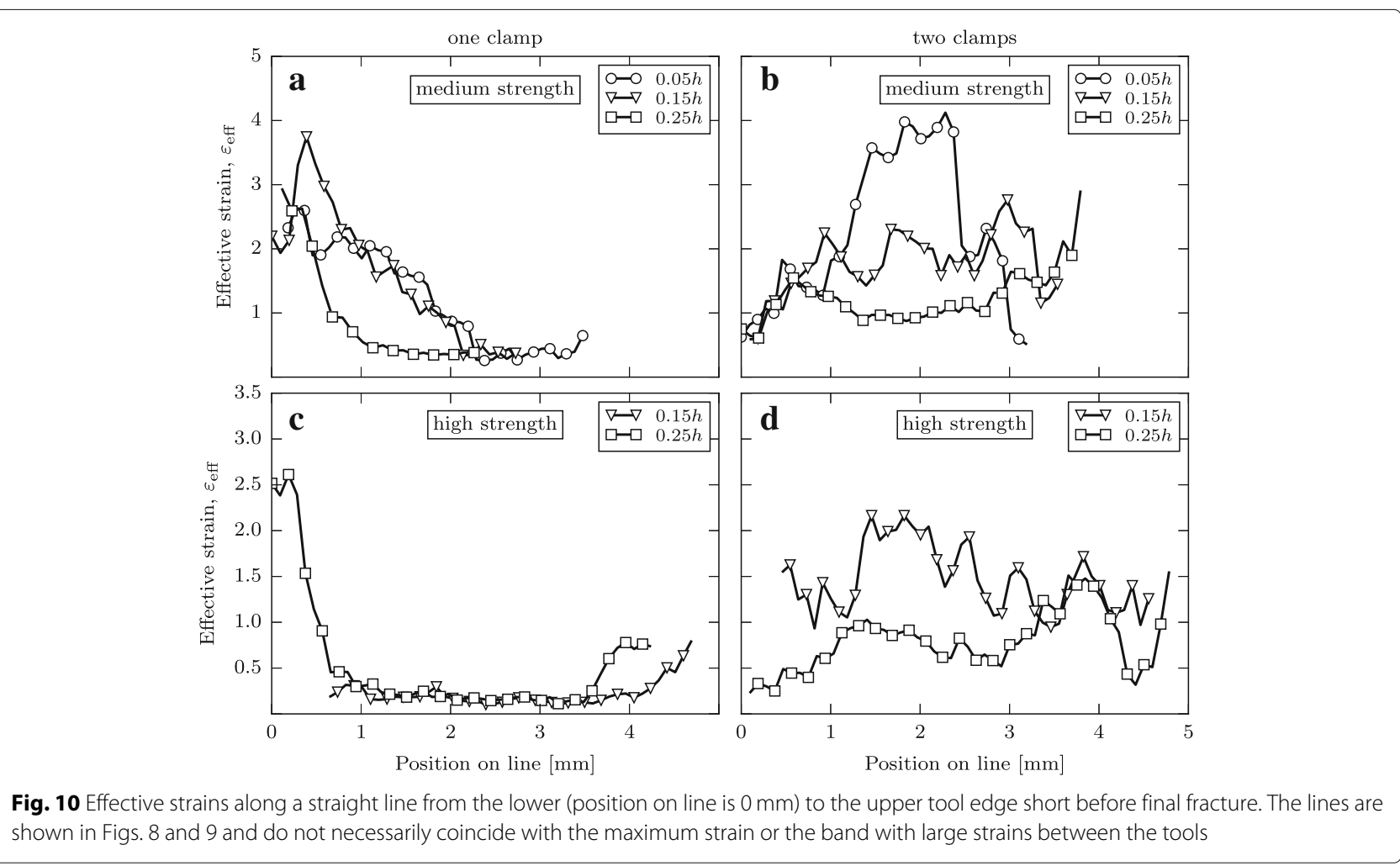


Table 3 Maximum strains at crack initiation $\bar{\varepsilon}_{\text {init }}$ and at final fracture $\bar{\varepsilon}_{\text {final }}$, and corresponding tool displacements, $\left|U_{y}\right|$ init $[\mathrm{mm}]$ and $\left|U_{y}\right|_{\text {final }}[\mathrm{mm}]$, respectively

\begin{tabular}{|c|c|c|c|c|c|c|c|c|c|c|}
\hline & \multicolumn{6}{|c|}{ Medium strength } & \multicolumn{4}{|c|}{ High strength } \\
\hline & \multicolumn{3}{|c|}{1 clamp } & \multicolumn{3}{|c|}{2 clamps } & \multicolumn{2}{|c|}{1 clamp } & \multicolumn{2}{|c|}{2 clamps } \\
\hline & $0.05 h$ & $0.15 h$ & $0.25 h$ & $0.05 h$ & $0.15 h$ & $0.25 h$ & $0.15 h$ & $0.25 h$ & $0.15 h$ & $0.25 h$ \\
\hline $\bar{\varepsilon}_{\text {init }}$ & 1.7 & 1.7 & 3.0 & 1.1 & 1.2 & 1.4 & 0.7 & 0.6 & 0.6 & 1.3 \\
\hline$\left|U_{y}\right|_{\text {init }}$ & 2.0 & 2.0 & 4.0 & 1.8 & 2.1 & 2.8 & 1.2 & 1.6 & 1.1 & 1.8 \\
\hline $\bar{\varepsilon}_{\text {final }}$ & 2.6 & 3.7 & 3.2 & 4.1 & 2.8 & 1.8 & 2.1 & 2.9 & 2.4 & 1.5 \\
\hline$\left|U_{y}\right|_{\text {final }}$ & 2.6 & 3.2 & 4.6 & 3.0 & 2.8 & 3.0 & 1.8 & 2.3 & 1.7 & 1.8 \\
\hline
\end{tabular}

clearance at a tool displacement of $1.6 \mathrm{~mm}$. Therefore, strains at a tool displacement of $1.6 \mathrm{~mm}$, for all sheared combinations for the medium and high strength materials, are shown in Figs. 14 and 15, respectively. Since the medium strength material fractured at a tool displacement much larger than $1.6 \mathrm{~mm}$, the strain fields for this material was also evaluated at a tool displacement of $2.6 \mathrm{~mm}$ and are shown in Fig. 16. In all cases, the strains at a given tool displacement were larger at small clearances than at large clearances. At $1.6 \mathrm{~mm}$ tool displacement the strains were considerably smaller in the medium strength material compared with the high-strength material due
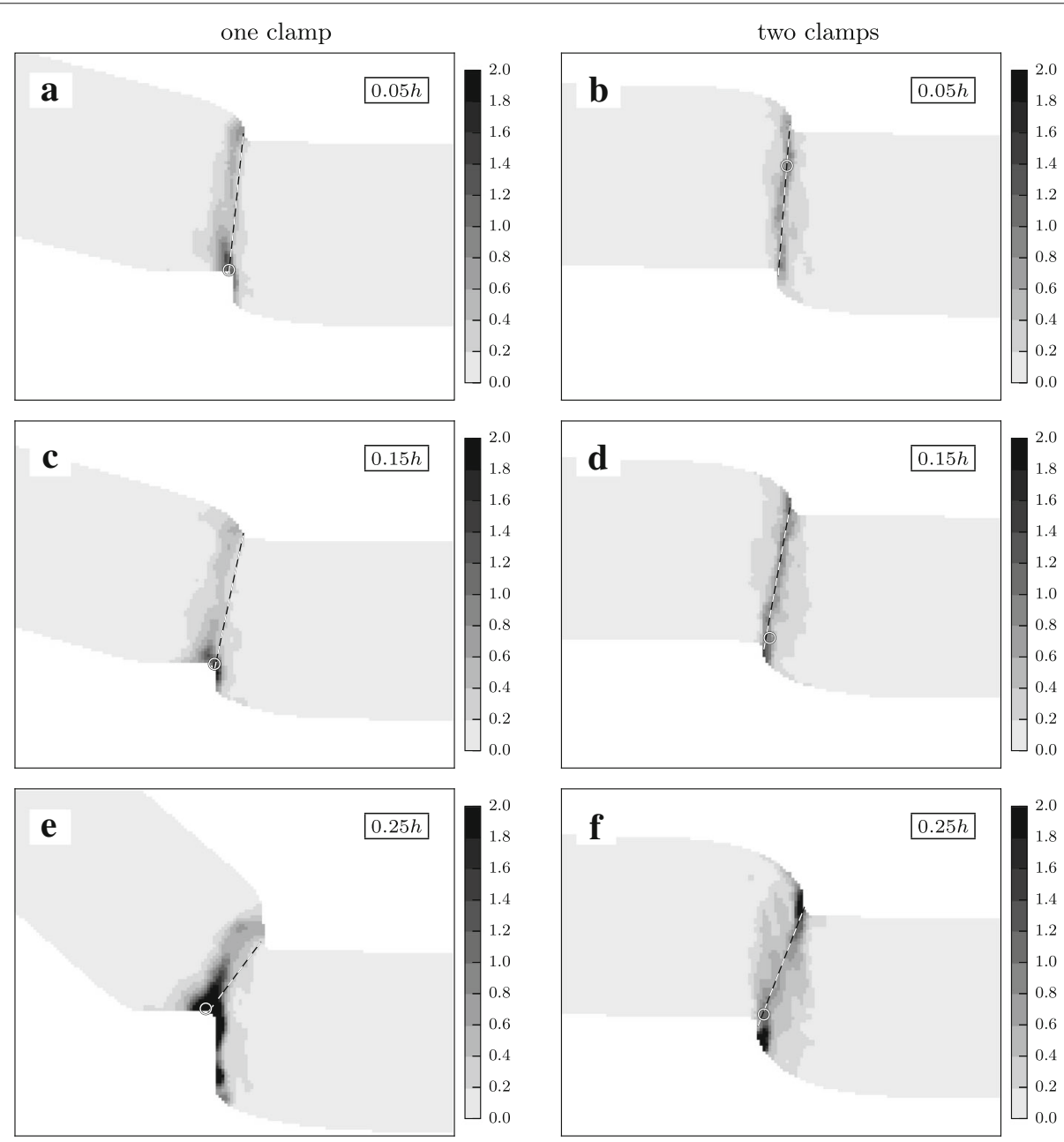

Fig. 11 Effective Cauchy strain fields at crack initiation for the medium strength material. Strains along the dashed black and white line are plotted in Fig. 13 and the maximum strains in the shear bands found inside the white circles are presented in Table 3 

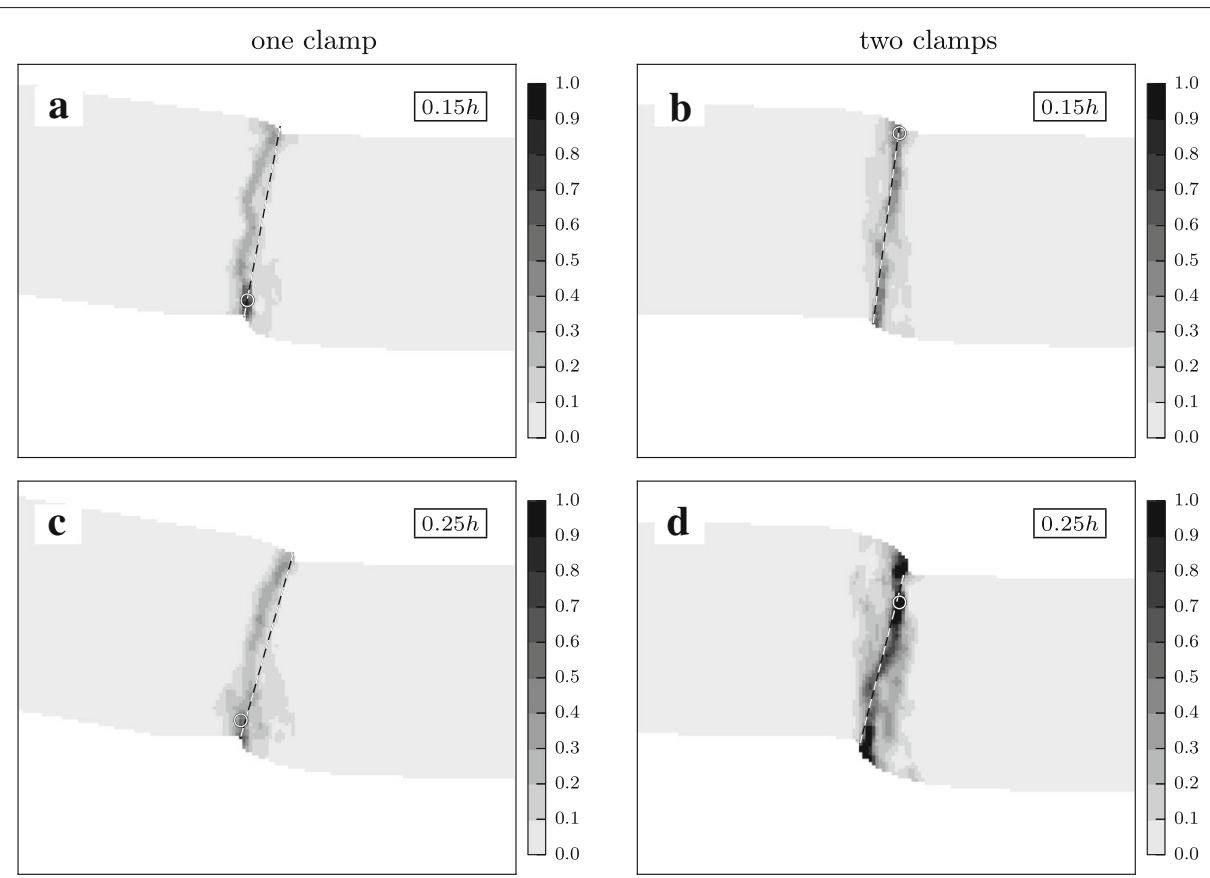

Fig. 12 Same as Fig. 11 but for the high-strength material

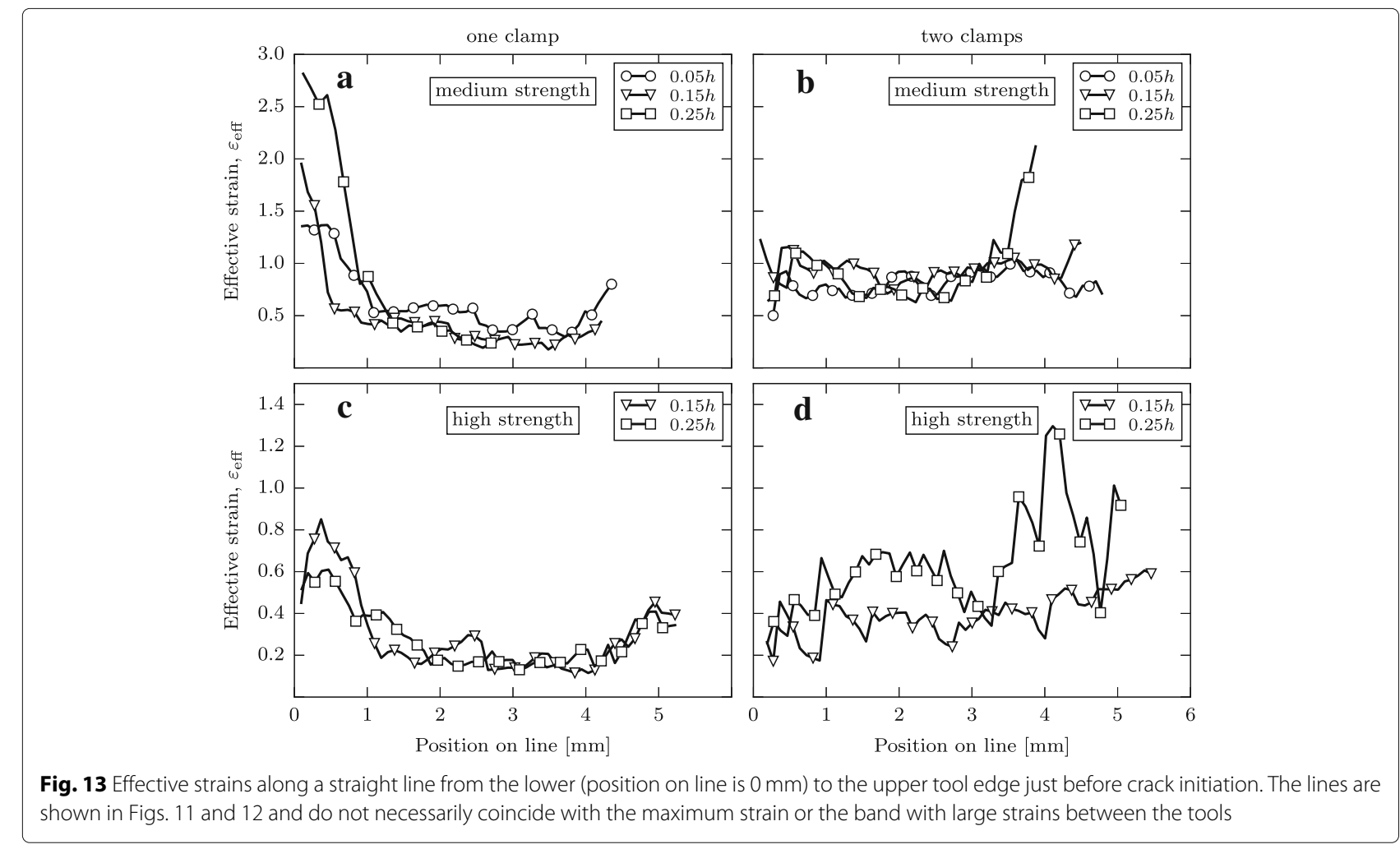




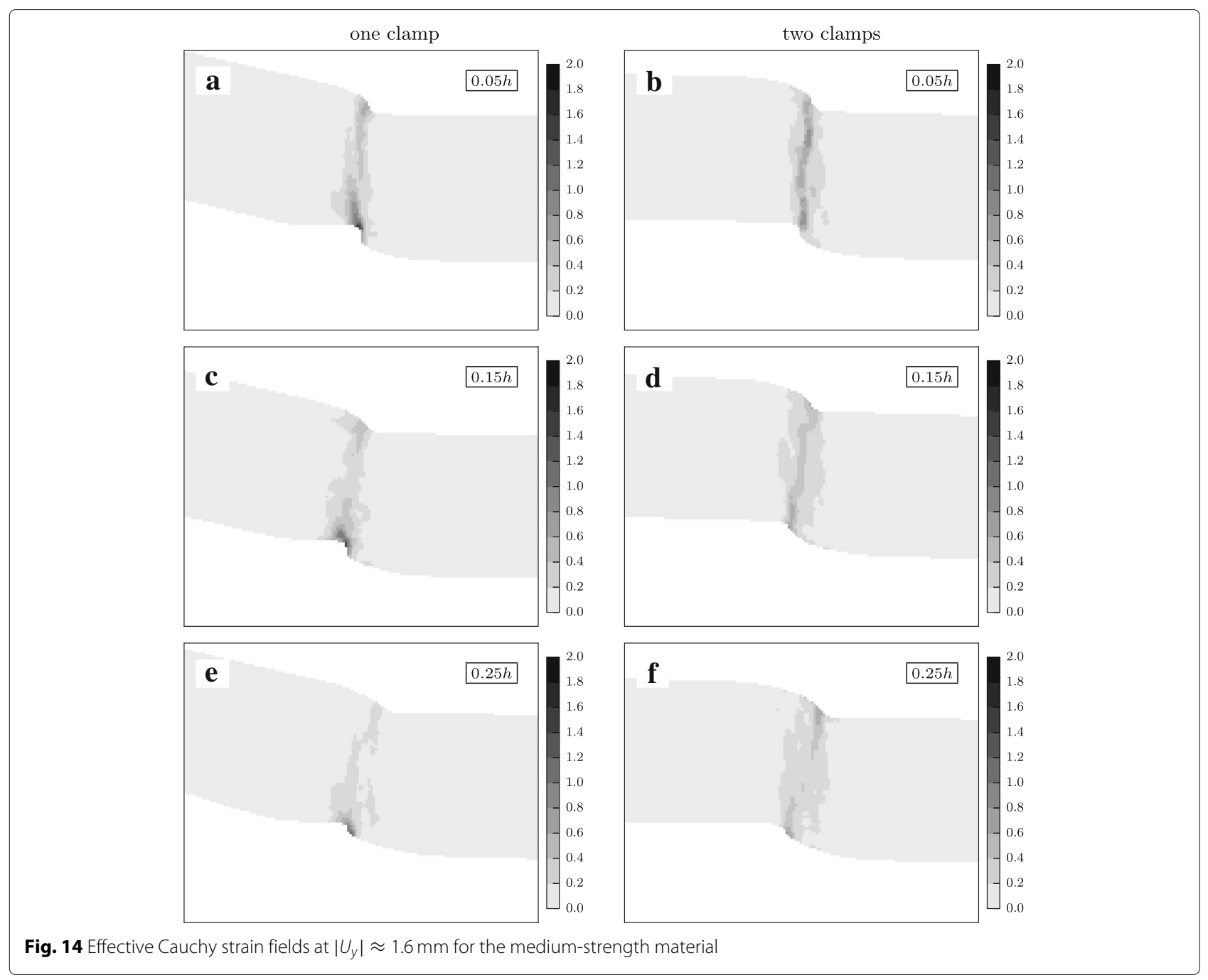

to a much wider strain band (larger deformed volume) in the medium-strength material. The medium-strength material is also more ductile and withstands larger strains before fracture.

\section{Discussion}

Comparison of effective strains from plane strain simulation, with strains on the strip surface in 3D simulations suggest that plane strain simulations are sufficient to describe the strain distribution in sheet metal shearing. The slightly larger strain in the middle of the sheared area seen in the plane strain plot (Fig. 1c) compared with the 3D strain plot (Fig. 1a and b) can be the result of inconsistent element sizes. The plane strain elements have half the size of the 3D elements and, thus, better resolve the large strain gradients.

The sheet samples were unintentionally delivered with bevelled edges $(0.2-0.3 \mathrm{~mm})$, which are seen as black borders between the speckled sheet surface and the tools in
Fig. 4a and b; in panel c the black border is thicker since unloading of elastic energy caused the fractured sheet to lift from the tool. Edge effects influence the result of the digital image correlation technique since some part of the analysed area extends outside the speckled surface. This bevel on the edges causes additional wasted information and can also locally affect the strains in the speckled surface since there is no contact between the tools and the bevelled edges. The purpose of the hole in the samples was to decrease the forces to similar levels as previously obtained during shearing in the experimental set-up (Gustafsson et al. 2016b; Gustafsson et al. 2016a) with a maintained image area and visibility of the imaged surface.

There was a good agreement between the Cauchy and Hencky strain tensors in all calculations. Furthermore, the final accumulated strains were relatively insensitive to variations in the number of strain increments. This number was chosen as a compromise between few increments with larger image distortion, and many increments 

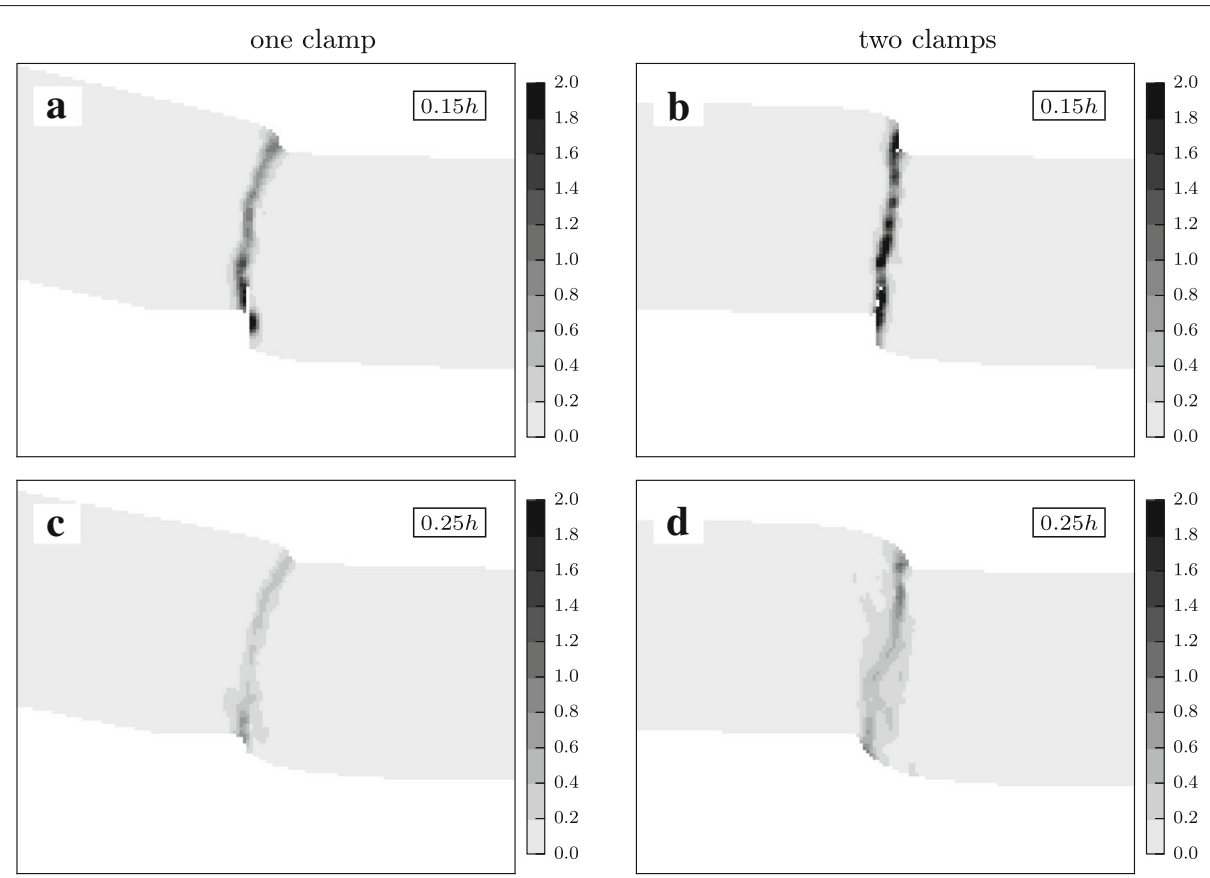

Fig. 15 Effective Cauchy strain fields at $\left|U_{y}\right| \approx 1.6 \mathrm{~mm}$ for the high-strength material

with more accumulated noise. The maximum number of increments was limited by the image capturing speed; the minimum number of increments was limited by the image correlation algorithm. With too few increments, there were unresolved areas around the narrow bands of largest strain due to high image distortion. The agreement between the Cauchy and Hencky strains indicate that the strain increments used were small enough for the accumulated strain to be close to the true strain.

In general, $\bar{\varepsilon}_{\text {final }}$ was larger than $\bar{\varepsilon}_{\text {init }}$ which means that the first part of the crack (close to the tool) is formed at a smaller strain than the strain needed for the last part of the crack propagation before final fracture. This can be the result of a varying stress state along the shear band, but, most probably, it is an effect of a limited resolution in the measurements. According to Dalloz et al. (2009), the depth of a heavily deformed zone is $0.2 \mathrm{~mm}$ when shearing a $1.5 \mathrm{~mm}$ thick sheet. That zone is probably larger in this study on $6 \mathrm{~mm}$ thick sheet, but still not much larger than the effective measurement length of $0.3 \mathrm{~mm}$. Therefore, both $\bar{\varepsilon}_{\text {init }}$ and $\bar{\varepsilon}_{\text {final }}$, but possibly $\bar{\varepsilon}_{\text {init }}$ to a larger extent, are probably underestimated in the measurements. Due to the nature of fracture, as an extremely local phenomenon, the resolution problem is inevitable, at least with the used technology. With knowledge of the resolution, however, the results are still useful.

Strains at the sheet edges that are in contact with the tools are in some extent influenced by relative movement in the contact region, since the interrogation windows at the sheet edges extends outside the sheet. The effects are largest at the vertical contact that is created during shearing, since the relative movement is largest there.

\section{Conclusions}

The digital image correlation (DIC) technique was used for reliable measurements of strains during sheet metal shearing. FE simulations show that strains on the sheet strip surface are comparable with strains inside the material and strains from a plane strain approximation. That observation extends the benefit of surface strain measurements as they are also relevant for the bulk material. The presented strains are useful for future validation of shearing models, and, coupled with a material model, to establish a fracture stress and to calibrate a fracture criteria. Some conclusions based directly on the strain measurements are:

- Most of the deformation in the sheet during shearing was concentrated to a "band" between the tools. The strain band was most straight at $0.15 \mathrm{~h}$ clearance. The band was, however, slightly thinner at $0.05 \mathrm{~h}$ clearance (medium strength material) especially when shearing with one clamp.

- With two clamps the strain band was almost symmetric, whereas with one clamp the strain band 

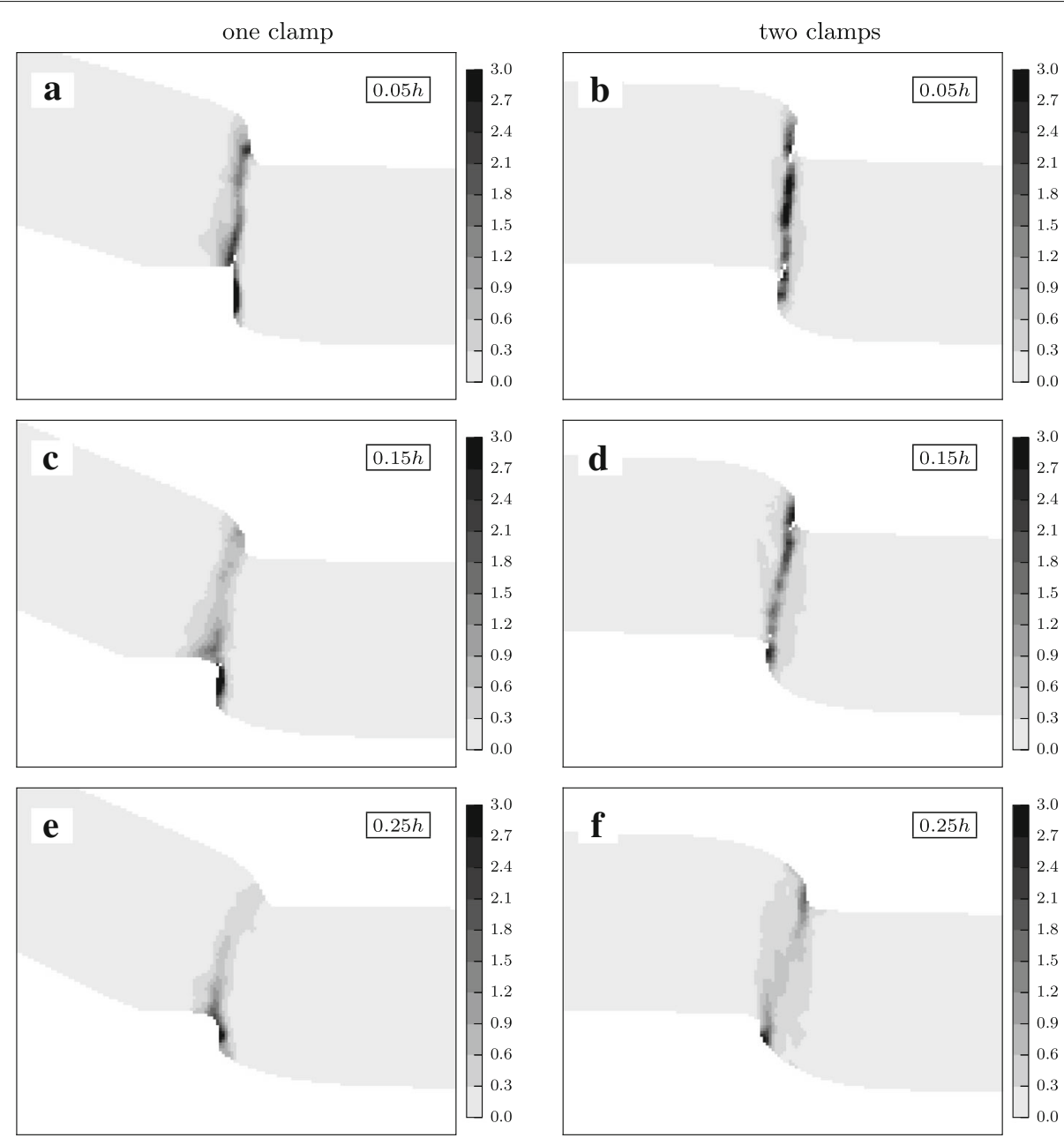

Fig. 16 Effective Cauchy strain fields at $\left|U_{y}\right| \approx 2.6 \mathrm{~mm}$ for the medium-strength material

was shifted towards the free strip end, and a large strain concentration was formed around the tool on the free side.

- Strains were larger in the high strength material compared with the medium strength material at the same tool displacement due to a wider strain band (larger deformed volume) in the medium strength material.

- The fracture strain was larger for the medium strength material compared with the high strength material and increased with increasing clearance.

\section{Acknowledgements}

This work was performed at Dalarna University within the Swedish Steel Industry Graduate School, with financial support from Högskolan Dalarna (Dalarna University), Jernkontoret (Swedish Steel Producers' Association), Länsstyrelsen i Gävleborg (County Administrative Board of Gävleborg), Region Dalarna (Regional Development Council of Dalarna), Region Gävleborg (Regional Development Council of Gävleborg), Sandvikens kommun (Municipality of Sandviken) and SSAB. Carl-Axel Norman is acknowledged for building the experimental set-up and preparing the material. SSAB provided the shearing tools and sheet metal samples.

\section{Authors' contributions}

EG conducted the experiments, collected the data, and wrote the major part of the paper. MO and LK are EG's PhD supervisors, who guided and supported this work and contributed with their expertise and advices. All authors read and approved the final manuscript.

\section{Competing interests}

The authors declare that they have no competing interests.

\section{Author details}

${ }^{1}$ Dalarna University, SE-791 88 Falun, Sweden. ${ }^{2}$ Luleå University of Technology, SE-971 87 Luleå, Sweden.

Received: 31 October 2016 Accepted: 21 November 2016

Published online: 08 December 2016

\section{References}

Crane, EV (1927). What happens in shearing metal. Machinery, 30, 225-230.

Dalloz, A, Besson, J, Gourgues-Lorenzon, AF, Sturel, T, Pineau, A (2009). Effect of shear cutting on ductility of a dual phase steel. Engineering Fracture Mechanics, 76(10), 1411-1424.

Gustafsson, E, Oldenburg, M, Jansson, A (2014). Design and validation of a sheet metal shearing experimental procedure. Journal of Materials Processing Technology, 214(11), 2468-2477. 
Gustafsson, E, Karlsson, L, Oldenburg, M (2016a). Experimental study of forces and energies during shearing of steel sheet with angled tools. International Journal of Mechanical and Materials Engineering, 11(1), 10. doi:10.1186/s40712-016-0063-1.

Gustafsson, E, Oldenburg, M, Jansson, A (2016b). Experimental study on the effects of clearance and clamping in steel sheet metal shearing. Journal of Materials Processing Technology, 229, 172-180.

Hollomon, JH (1945). Tensile deformation. Transactions of the American institute of mining and metallurgical engineers, 162, 268-290.

Izod, EG (1906). Behaviour of materials of construction under pure shear. Proceedings of The Institution of Mechanical Engineers, 70, 5-55.

Kopp, T, Stahl, J, Demmel, P, Tröber, P, Golle, R, Hoffmann, H, Volk, W (2016). Experimental investigation of the lateral forces during shear cutting with an open cutting line. Journal of Materials Processing Technology, 238, 49-54.

Stegeman, YW, Goijaerts, AM, Brokken, D, Brekelmans, WAM, Govaert, LE, Baaijens, FPT (1999). An experimental and numerical study of a planar blanking process. Journal of Materials Processing Technology, 87, 266-276.

Sutton, MA, Orteu, JJ, Schreier, H (2009). Image correlation for shape, motion and deformation measurements: Springer. ISBN:0387787461.

Tarigopula, V, Hopperstad, OS, Langseth, M, Clausen, AH, Hild, F, Lademo, OG, Eriksson, M (2008). A study of large plastic deformations in dual phase steel using digital image correlation and FE analysis. Experimental Mechanics, 48(2), 181-196.

Weaver, HP, \& Weinmann, KJ (1985). A study of edge characteristics of sheared and bent steel plate. Journal of Applied Metalworking, 3(4), 381-390.

Wu, X, Bahmanpour, H, Schmid, K (2012). Characterization of mechanically sheared edges of dual phase steels. Journal of Materials Processing Technology, 212, 1209-1224.

Yamasaki, S, \& Ozaki, T (1991). Shearing of inclined sheet metals: Effect of inclination angle. JSME international journal Ser 3, Vibration, control engineering, engineering for industry, 34(4), 533-539.

\section{Submit your manuscript to a SpringerOpen ${ }^{\circ}$ journal and benefit from:}

- Convenient online submission

- Rigorous peer review

- Immediate publication on acceptance

- Open access: articles freely available online

High visibility within the field

- Retaining the copyright to your article

Submit your next manuscript at $\boldsymbol{s p r i n g e r o p e n . c o m ~}$ 